\title{
In vitro and in vivo comparison of transport media for detecting nasopharyngeal carriage of Streptococcus pneumoniae
}

\author{
Anneke Steens ${ }^{\text {Corresp., }}{ }^{1,2}$ ， Natacha Milhano ${ }^{1,3}$ ， Ingeborg S Aaberge ${ }^{1}$ ， Didrik Frimann Vestrheim ${ }^{1}$ \\ 1 Infection Control and Environmental Health, Norwegian Institute of Public Health, Oslo, Norway \\ 2 Faculty of Medicine, University of Oslo, Oslo, Norway \\ 3 European Programme for Public Health Microbiology Training (EUPHEM), European Centre for Disease Prevention and Control (ECDC), Stockholm, \\ Sweden \\ Corresponding Author: Anneke Steens \\ Email address: anneke.steens@fhi.no
}

Background As standard method for pneumococcal carriage studies the World Health Organization recommends nasopharyngeal swabs be transported and stored at cool temperatures in a medium containing skim-milk, tryptone, glucose and glycerol (STGG). An enrichment broth used for transport at room temperature in three carriage studies performed in Norway may have a higher sensitivity than STGG. We therefore compared the media in vitro and in vivo. Methods For the in vitro component, three strains (serotype 4, 19F and 3) were suspended in STGG and enrichment broth. Recovery was compared using latex agglutination, quantification of bacterial loads by real-time PCR of the lytA gene, and counting colonies from incubated plates. For the in vivo comparison, paired swabs were obtained from 100 children and transported in STGG at cool temperatures or in enrichment broth at room temperature. Carriage was identified by latex agglutination and confirmed by Quellung reaction. Results In vitro, the cycle threshold values obtained by PCR did not differ between the two media $(p=0.853)$ and no clear difference in colony counts was apparent after incubation ( $p=0.593)$. In vivo, pneumococci were recovered in $46 \%$ of swabs transported in STGG and $51 \%$ of those transported in enrichment broth (Kappa statistic 0.90, $\mathrm{p}=0.063$ ). Discussion Overall, no statistical differences in sensitivity were found between STGG and enrichment broth. Nevertheless, some serotype differences were observed and STGG appeared slightly less sensitive than enrichment broth for detection of nasopharyngeal carriage of pneumococci by culturing. We recommend the continued use of STGG for transport and storage of nasopharyngeal swabs in pneumococcal carriage studies for the benefit of comparability between studies and settings, including more resource-limited settings. 
1 In vitro and in vivo comparison of transport media for detecting

2 nasopharyngeal carriage of Streptococcus pneumoniae

3

4 Running title: pneumococcus media comparison

5

6 Anneke Steens $^{1,2}$, Natacha Milhano ${ }^{1,3}$, Ingeborg S Aaberge ${ }^{1}$, Didrik F Vestrheim ${ }^{1}$

7

8 Affiliations:

$9{ }^{1}$ Infection Control and Environmental Health, Norwegian Institute of Public Health, Oslo,

10 Norway

$12^{2}$ Faculty of Medicine, University of Oslo, Oslo, Norway

$12{ }^{3}$ European Programme for Public Health Microbiology Training (EUPHEM), European Centre

13 for Disease Prevention and Control (ECDC), Stockholm, Sweden

14

15 Corresponding author: Anneke Steens: anneke.steens@,fhi.no

16

17 
18 Abstract

\section{Background}

As standard method for pneumococcal carriage studies the World Health Organization recommends nasopharyngeal swabs be transported and stored at cool temperatures in a medium containing skim-milk, tryptone, glucose and glycerol (STGG). An enrichment broth used for transport at room temperature in three carriage studies performed in Norway may have a higher sensitivity than STGG. We therefore compared the media in vitro and in vivo.

\section{Methods}

For the in vitro component, three strains (serotype 4, 19F and 3) were suspended in STGG and enrichment broth. Recovery was compared using latex agglutination, quantification of bacterial loads by real-time PCR of the lytA gene, and counting colonies from incubated plates. For the in vivo comparison, paired swabs were obtained from 100 children and transported in STGG at cool temperatures or in enrichment broth at room temperature. Carriage was identified by latex agglutination and confirmed by Quellung reaction.

\section{Results}

In vitro, the cycle threshold values obtained by PCR did not differ between the two media $(p=0.853)$ and no clear difference in colony counts was apparent after incubation $(p=0.593)$. In vivo, pneumococci were recovered in $46 \%$ of swabs transported in STGG and $51 \%$ of those transported in enrichment broth (Kappa statistic 0.90, $\mathrm{p}=0.063$ ).

\section{Discussion}

Overall, no statistical differences in sensitivity were found between STGG and enrichment broth. Nevertheless, some serotype differences were observed and STGG appeared slightly less sensitive than enrichment broth for detection of nasopharyngeal carriage of pneumococci by culturing.

We recommend the continued use of STGG for transport and storage of nasopharyngeal swabs in pneumococcal carriage studies for the benefit of comparability between studies and settings, including more resource-limited settings. 


\section{Introduction}

Monitoring carriage of Streptococcus pneumoniae (pneumococci) is important for determining changes after vaccine introduction in national immunisation programmes. To enable comparison of results from different studies and countries, the World Health Organization Pneumococcal Carriage Working Group published a set of standard methods for such studies measuring nasopharyngeal carriage of pneumococci [1]. A medium containing skim milk powder, tryptone soy broth, glucose and glycerol in distilled water (STGG) is recommended for transport and storage of nasopharyngeal specimens, and transport should be done at cool temperatures [2]. Studies using STGG in developed countries have generally revealed prevalences of pneumococcal carriage in children of around 30-50\% [3-5]. In Norway, several carriage studies have been performed using enrichment broth (beef infusion enriched with 5\% horse serum and 3.3\% defibrinated horse blood [6]; Statens Serum Institute, Copenhagen, Denmark) for transport at room temperature. Carriage prevalence in those studies was around $80 \%$ before and after introduction of the 7-valent pneumococcal conjugate vaccine (PCV), and 62\% two years after switching to the 13-valent PCV [7]. Although different factors may contribute to this high prevalence, such as the percentage of children in day-care (>90\% [8]) and the low use of antibiotics in Norway $[9,10]$, results suggest that enrichment broth transported at room temperature may be more sensitive for detection of carriage than STGG transported at cool temperatures.

We therefore compared 1) in vitro recovery from serial dilutions in STGG and enrichment broth and 2) in vivo detection of nasopharyngeal carriage of pneumococci from swabs that were transported and stored in STGG at cool temperatures or in enrichment broth at room temperature.

\section{Materials and methods}

In the in vitro component of the study we compared recovery rates of pneumococci from serial dilutions that had been stored at different temperatures and media using I) culturing, II) a commercial latex agglutination kit and III) quantitative real-time PCR (qPCR). In the in vivo 
component we compared carriage in paired swabs taken from children attending day-care centres and transported in STGG or enrichment broth. In this second part we used methods I and II for detection of pneumococci. See Figure 1 for a schematic overview of the procedures. Note that we followed the recommended conditions for transport and storage for each medium (wet ice/cool box for STGG and freezing [in vivo only], room temperature and immediate processing for enrichment broth).

\section{In vitro comparison}

Three strains belonging to different serotypes were used as pneumococcal samples; two reference strains (ATCC 49619 - serotype 19F, and TIGR4 - serotype 4), and a strain belonging to serotype 3 obtained from the 2013 sample of a previous Norwegian carriage study [7]. Colonies from each serotype were suspended in Todd-Hewitt (TH) broth at a concentration of $0.5 \mathrm{McFarland}$ in serial 1:10 dilutions of $10^{-2}$ to $10^{-5}$ (minimal concentration for which recovery of pneumococcal DNA was possible; see online-only document). A volume of $100 \mu \mathrm{L}$ of serotype dilution in TH broth was added to each set of transport media (either $1 \mathrm{ml}$ of STGG or $3 \mathrm{ml}$ of enrichment broth) to prepare the pneumococcal samples. The samples were left for $3 \mathrm{~h}$ on wet ice (STGG) or room temperature (enrichment broth). Subsequently, $100 \mu \mathrm{L}$ of the samples was plated on Columbia horse blood agar plates. Furthermore, $100 \mu \mathrm{L}$ of the STGG samples was added to $3 \mathrm{ml}$ fresh enrichment broth. All was done in triplicate. Plates and tubes (broth sample made from the STGG samples and the initial enrichment broth samples) were incubated overnight at $35^{\circ} \mathrm{C}$ with $5 \% \mathrm{CO}_{2}$.

Pneumococci were identified by latex agglutination (Pneumotest-Latex kit [11]; Statens Serum Institut, Denmark) from the incubated broths. Quantification of the bacterial loads was performed by qPCR (see below for details) and counting of the colony forming units (CFU) from the incubated plates. 
100 From each sample $200 \mu \mathrm{L}$ was boiled for 10 minutes and DNA was extracted by QIAamp DNA

101 Mini QIAcube kit (Qiagen, Inc., Valencia, CA, US) according to the manufacturer's

102 recommendations. A qPCR assay for the detection of the autolysin-encoding gene (lytA) was

103 then performed as described before by Carvalho et al. [12]. Briefly, 25 $\mu \mathrm{L}$ reaction volume

104 composed of TaqMan Fast Universal PCR Master Mix (2x), 200 nM of each primer and probe,

105 10x Exo IPC-mix, 50x Exo IPC DNA and $2 \mu \mathrm{L}$ of DNA was run at $50^{\circ} \mathrm{C}$ for 2 minutes,

106 denaturation at $95^{\circ} \mathrm{C}$ for 10 minutes, followed by 40 amplification cycles of $95^{\circ} \mathrm{C}$ for 15 seconds

107 and $60^{\circ} \mathrm{C}$ for 1 minute. Samples were considered negative if cycle thresholds $(\mathrm{Ct})$ exceeded 40.

108 A positive (ATCC49619) and a non-template control (sterile water) were included in each run,

109 along with extraction controls.

In vivo comparison

112 This comparison was performed as part of a larger carriage study (sample taken in 2015 [13]).

113 The study was conducted in accordance with principles of the Declaration of Helsinki, and

114 approved by the Regional Committee for Medical Research Ethics, South-Eastern Norway

115 (reference number: 2014/2046). Parents/guardians of the children gave written informed consent

116 before including their child in the study. The study design resembles the design used in previous

117 Norwegian carriage studies [7].

118 Two flocked nylon nasopharyngeal swabs (E-swabsTM, Copan, Italy) taken from the same 119 nostril were collected from 100 children aged 1-6 years according to standard procedures. The

120 first swab was placed in $1 \mathrm{ml}$ STGG which was subsequently stored in a cool box and the second

121 swab was stored and transported in $3 \mathrm{ml}$ enrichment broth at room temperature. The specimens

122 were processed within 4 hours of sampling. The STGG samples were vortexed at high speed and

123 frozen at $-70^{\circ} \mathrm{C}$, following the recommendations of WHO [1]. Within one month but earliest

124 after 18 hours after initial freezing, the STGG samples were processed further: after being

125 thawed and vortexed, $200 \mu 1$ was added to fresh enrichment broth and $20 \mu 1$ was plated on

126 gentamycin-blood-agar (GBA). The swabs from the enrichment broth samples were plated on

127 GBA within 4 hours of sampling and the swab was re-inserted into the enrichment broth. All

128 broths and GBA plates were incubated overnight at $35^{\circ} \mathrm{C}$, with $5 \% \mathrm{CO}_{2}$. 
129 Pneumococci were identified by latex agglutination from incubated broths. Confirmation and 130 factor typing were performed by Quellung reaction. All morphologically different pneumococcal 131 colonies per plate were typed. In cases where the latex agglutination was positive but no colonies 132 were found on plates after incubation overnight, samples were re-cultured by plating one drop of 133 the incubated broth and incubating this for another night before further analysis.

Data analysis

136 For the in vitro analyses, $\mathrm{Ct}$ values and CFU counts (after a logarithmic transformation; $\log \mathrm{CFU}$ ) 137 from the two media were compared by linear regression. We used 11 mutually exclusive dummy

138

139

variables identifying different dilution-serotype combinations, which enabled us to simultaneously run the comparison for all serotypes. Additionally, the analyses were conducted separately per serotype.

Agreement in the in vivo comparison was determined using the kappa statistic [14] and the exact McNemar's probability test for paired data. Data were analysed in Stata 14.0 and GraphPad Prism 5.

\section{Results}

In vitro results

The latex agglutination test was positive for both media for all serotype dilutions tested, with the exception of serotype 3 at a dilution of $10^{-5}$ in STGG, where no pneumococci were detected.

The quantification of DNA by $\mathrm{Ct}$ value is presented in Figure 2. The $\mathrm{Ct}$ values overall did not differ significantly between STGG and enrichment broth samples ( $p=0.853)$, though for serotype 4, the Ct values were significantly lower for STGG samples compared to enrichment broth samples ( $\mathrm{p}=0.007)$. After culturing, no clear overall difference was found in the $\log C F U$ $(p=0.593)$ but significant differences were observed for serotype 4 (Figure 3B; $p=0.008$; more colonies on plates incubated with STGG samples) and serotype 3 (Figure $3 \mathrm{C} ; \mathrm{p}=0.001$; more colonies on plates incubated with enrichment broth samples). 
157 In vivo results

158 Forty-six percent of the swabs transported and stored in STGG and 51\% of those transported in 159 enrichment broth were positive for pneumococci. This resulted in a Kappa statistic for carriage 160 of 0.90 for the paired swabs (Table 1), indicating a trend towards higher sensitivity after 161 transportation in enrichment broth compared to STGG $(\mathrm{p}=0.0625)$. If re-cultured samples were 162 excluded, carriage was 44\% for the samples transported and stored in STGG and 48\% for those 163 transported in enrichment broth (Kappa $=0.92 ; \mathrm{p}=0.125)$. For each child for whom both swabs 164 were positive, the same serotype was obtained. In one child, carriage of two serotypes was found 165 in the enrichment broth sample, while one serotype was found in the STGG sample. However, 166 the plate incubated with the enrichment broth sample had only one colony of the serotype that 167 was missed in the STGG sample (serotype 3) and the agglutination test was negative for this 168 serotype, indicating presence at a very low concentration.

\section{Discussion}

Overall, no statistical differences in sensitivity were found between STGG stored and transported at cool temperatures and enrichment broth transported at room temperature. Nevertheless, some serotype differences were observed as well as a trend towards higher sensitivity for detection of pneumococcal carriage after transportation in enrichment broth compared to STGG.

There are several possible reasons for these differences. In vitro, a pure dilution of one serotype was used in place of a nasopharyngeal swab. However, in vivo the swab contained different

177 respiratory bacteria and viruses, and was covered with mucus and cellular debris. The presence

178 of other bacteria places pneumococci in competition for nutrients needed for growth and 179 reproduction. These nutrients are available in higher concentration in enrichment broth than in 180 STGG, which may explain the small difference in sensitivity observed between the in vitro and in vivo settings. In addition to the difference in available nutrients in STGG and enrichment

182 broth, the fact that STGG samples were kept in a cool box during transport while the enrichment 183 broth samples were kept at room temperature, may have caused the small non-significant 
184 difference in carriage prevalence. The original carriage study presenting the use of enrichment

185 broth as transport medium used cool transport conditions [6] but three previous Norwegian 186 carriage studies used room temperature [7]. The present study is not designed to differentiate

187 between the effect of media and temperature, but while pneumococci are thought to thrive better 188 at warmer temperatures, we did not find an overall difference between the methods in bacterial 189 load or DNA quantity in vitro. Whether the relative abundance of other respiratory bacteria / the 190 microbiome may have changed and would no longer be representative of the nasopharyngeal 191 tract after storage at room temperature should be investigated were room temperature to be used 192 in such studies. Still, for serotype 3, the bacterial load indicated a higher sensitivity of enrichment broth compared to STGG. The serotype 3 isolate was obtained from a previous carriage study. For serotype $19 \mathrm{~F}$ and 4 , reference strains were used. The origin of the isolates (reference strains versus carriage isolate) may have induced different bacterial growth characteristics. Further, the capsule structure differs between serotypes (serotype 3 being very mucoid). The low number of serotypes tested and the difference in origin between serotypes are limitations to the in vitro part of this study.

199 The carriage prevalence found in this study is lower than observed previously in Norway [7], and 200 more similar to what has been seen in other developed countries [3-5]. The methods used for swab collection, transport and incubation in enrichment broth and culturing were unchanged

202 from former studies, indicating a real difference in carriage prevalence that may have resulted 203 from vaccination.

204 Advantages of molecular based techniques compared to culture techniques include the fact that 205 viable organisms are not required, the original composition of the nasopharyngeal specimen is 206 preserved, and detailed quantification and characterization of the pneumococci within a sample 207 are possible, depending on the methods used [1]. Furthermore, the sensitivity of molecular 208 methods for detection of multiple co-colonising serotypes has been shown to be higher than 209 conventional methods [15]. Nevertheless, isolation of strains enables further characterization 210 such as antimicrobial susceptibility testing and sequence typing, and should not be replaced by 211 molecular methods alone, despite its high sensitivity. The additional step including latex 212 agglutination appears to be of value for identification and isolation of pneumococci from 
213 multiple carriage [6]. The PneuCarriage Project concluded that microarray with a culture

214 amplification step has the highest sensitivity for determining carriage [16].

215 Finally, STGG is cheap, easy to make and can be stored longer than enrichment broth, thus

216 enabling comparability between studies and settings, including more resource-limited settings.

217 Furthermore, STGG transported and stored at cool temperatures enables studies to investigate the

218 microbiome $[17,18]$, whereas enrichment broth may selectively stimulate growth of

219 pneumococci. Therefore, even though STGG appeared slightly less sensitive than enrichment

220 broth for detection of nasopharyngeal carriage of pneumococci by culturing, we recommend the

221 continued use of STGG for transport and storage of nasopharyngeal swabs at cool temperatures

222 in future carriage studies.

\section{Acknowledgements}

225 We thank the children and parents for participating and day-care centres workers for their 226 support. We also thank Anne Ramstad Alme, Gunnhild Rødal, and Lene Haakensen for the 227 laboratory analyses, Ingvild Essén and Kristine Hartmark for the collection of nasopharyngeal 228 swabs, and Richard White for statistical advice.

\section{References}

1. Satzke, C., P. Turner, A. Virolainen-Julkunen, P.V. Adrian, M. Antonio, K.M. Hare, A.M. HenaoRestrepo, A.J. Leach, K.P. Klugman, B.D. Porter, R. Sa-Leao, J.A. Scott, H. Nohynek, K.L. O'Brien, and W.H.O.P.C.W. Group, Standard method for detecting upper respiratory carriage of Streptococcus pneumoniae: updated recommendations from the World Health Organization Pneumococcal Carriage Working Group. Vaccine, 2013. 32(1): p. 165-79.

2. O’Brien, K.L., M.A. Bronsdon, R. Dagan, P. Yagupsky, J. Janco, E. J, C.G. Whitney, Y.-H. Yang, L.G.E. Robinson, B. Schwartz, and G.M. Carlone, Evaluation of a medium (STGG) for transport and optimal recovery of Streptococcus pneumoniae from nasopharyngeal secretions collected during field studies. J Clin Microbiol, 2001. 39(3): p. 1021-1024.

3. van Hoek, A.J., C.L. Sheppard, N.J. Andrews, P.A. Waight, M.P. Slack, T.G. Harrison, S.N. Ladhani, and $\mathrm{E}$. Miller, Pneumococcal carriage in children and adults two years after introduction of the thirteen valent pneumococcal conjugate vaccine in England. Vaccine, 2014. 32: p. 4349-4355.

4. Desai, A.P., D. Sharma, E.K. Crispell, W. Baughman, S. Thomas, A. Tunali, L. Sherwood, A. Zmitrovich, R. Jerris, S.W. Satola, B. Beall, M.R. Moore, S. Jain, and M.M. Farley, Decline in Pneumococcal Nasopharyngeal Carriage of Vaccine Serotypes After the Introduction of the 13- 
Valent Pneumococcal Conjugate Vaccine in Children in Atlanta, Georgia. Pediatr Infect Dis J, 2015. 34(11): p. 1168-74.

5. Andrade, A.L., Y.M. Ternes, M.A. Vieira, W.G. Moreira, J. Lamaro-Cardoso, A. Kipnis, M.R. Cardoso, M.C. Brandileone, I. Moura, F.C. Pimenta, M. da Gloria Carvalho, F.O. Saraiva, C.M. Toscano, and R. Minamisava, Direct effect of 10-valent conjugate pneumococcal vaccination on pneumococcal carriage in children Brazil. PLoS One, 2014. 9(6): p. e98128.

6. Kaltoft, M.S., U.B. Skov Sorensen, H.C. Slotved, and H.B. Konradsen, An easy method for detection of nasopharyngeal carriage of multiple Streptococcus pneumoniae serotypes. J Microbiol Methods, 2008. 75(3): p. 540-4.

7. Steens, A., D.A. Caugant, I.S. Aaberge, and D.F. Vestrheim, Decreased Carriage and Genetic Shifts in the Streptococcus pneumoniae Population After Changing the 7-Valent to the 13-Valent Pneumococcal Vaccine in Norway. Pediatr Infect Dis J, 2015. 34: p. 875-883.

8. Statistics Norway, Child-care, 2010 [in Norwegian: Barn i barnehage - foreldrebakgrunn og utvikling de seneste årene]. ISBN: 978-82-537-7794-8 (electronic) 2010.

9. Garcia-Rodriguez, J.A. and M.J. Fresnadillo Martinez, Dynamics of nasopharyngeal colonization by potential respiratory pathogens. J Antimicrob Chemother, 2002. 50 Suppl S2: p. 59-73.

10. NORM-VET, N., Usage of antimicrobial agents and occurence of antimicrobial resistance in Norway. ISSN:1502-2307 (print) / 1890-9965 (electronic) 2014.

11. Slotved, H.-C., M. Kaltoft, I.C. Skovsted, M.B. Kerrn, and F. Espersen, Simple, Rapid Latex Agglutination Test for Serotyping of Pneumococci (Pneumotest-Latex). J Clin Microbiol, 2004. 42: p. 2518-2522.

12. Carvalho Mda, G., M.L. Tondella, K. McCaustland, L. Weidlich, L. McGee, L.W. Mayer, A. Steigerwalt, M. Whaley, R.R. Facklam, B. Fields, G. Carlone, E.W. Ades, R. Dagan, and J.S. Sampson, Evaluation and improvement of real-time PCR assays targeting lyt $A$, ply, and psaA genes for detection of pneumococcal DNA. J Clin Microbiol, 2007. 45(8): p. 2460-6.

13. Steens A , C.D., Aaberge IS, Vestrheim DF, Continued decrease in pneumococcal carriage after implementation of pcv13 in norway. Abstract 370. 10th International Symposium on Pneumococci and Pneumococcal Diseases (ISPPD 2016). Glasgow, Scotland, from 26-30 June, 2016, 2016.

14. Landis, J.R. and G.G. Koch, The measurement of observer agreement for categorical data. Biometrics, 1977. 33(1): p. 159-74.

15. Saha, S., J.K. Modak, H. Naziat, H.M. Al-Emran, M. Chowdury, M. Islam, B. Hossain, G.L. Darmstadt, C.G. Whitney, and S.K. Saha, Detection of co-colonization with Streptococcus pneumoniae by algorithmic use of conventional and molecular methods. Vaccine, 2015. 33(5): p. 713-8.

16. Satzke, C., E.M. Dunne, B.D. Porter, K.P. Klugman, E.K. Mulholland, and g. PneuCarriage project, The PneuCarriage Project: A Multi-Centre Comparative Study to Identify the Best Serotyping Methods for Examining Pneumococcal Carriage in Vaccine Evaluation Studies. PLoS Med, 2015. 12(11): p. e1001903.

17. Grijalva, C.G., M.R. Griffin, K.M. Edwards, M. Johnson, A.I. Gil, H. Verastegui, C.F. Lanata, and J.V. Williams, Concordance between RT-PCR-based detection of respiratory viruses from nasal swabs collected for viral testing and nasopharyngeal swabs collected for bacterial testing. J Clin Virol, 2014. 60(3): p. 309-12.

18. Turner, P., L. Po, C. Turner, D. Goldblatt, and F. Nosten, Detection of respiratory viruses by PCR assay of nasopharyngeal swabs stored in skim milk-tryptone-glucose-glycerol transport medium. J Clin Microbiol, 2011. 49(6): p. 2311-3. 
293 Tables

294 Table 1: Pneumococcal carriage determined by culturing paired nasopharyngeal swabs stored 295 in STGG or enrichment broth

\begin{tabular}{ccccc}
\hline & & \multicolumn{2}{c}{ Enrichment broth } & \\
\cline { 3 - 4 } STGG & Positive & 46 & Negative & Total \\
\cline { 3 - 4 } & Negative & 5 & 0 & 46 \\
& Total & 51 & 49 & 54 \\
& & 49 & 100 \\
\hline
\end{tabular}




\section{Figures}

298 Figure 1: Schematic overview of the experimental designs. Left: in vitro. Right: in vivo.

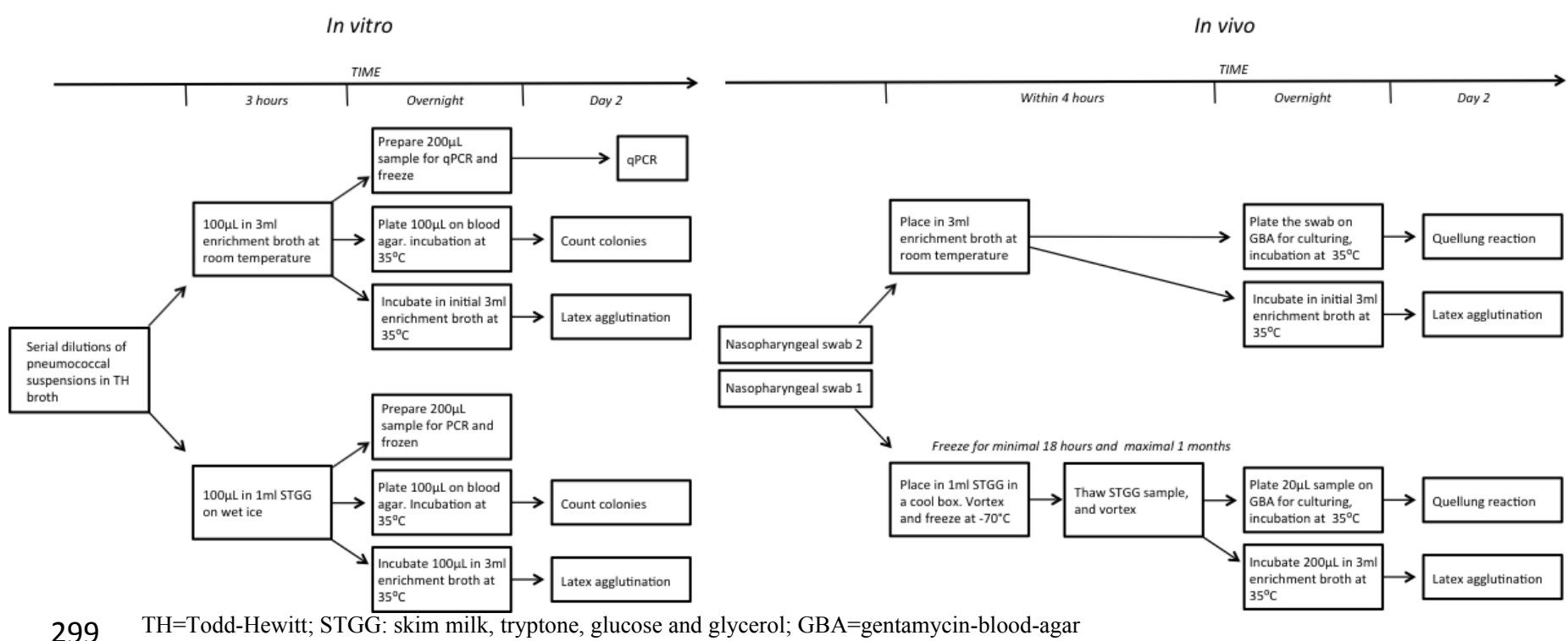


300 Figure 2: DNA quantification (Ct values) at different dilutions of enrichment broth and STGG.

301 A: serotype 19F*; B: serotype 4; C: serotype 3.
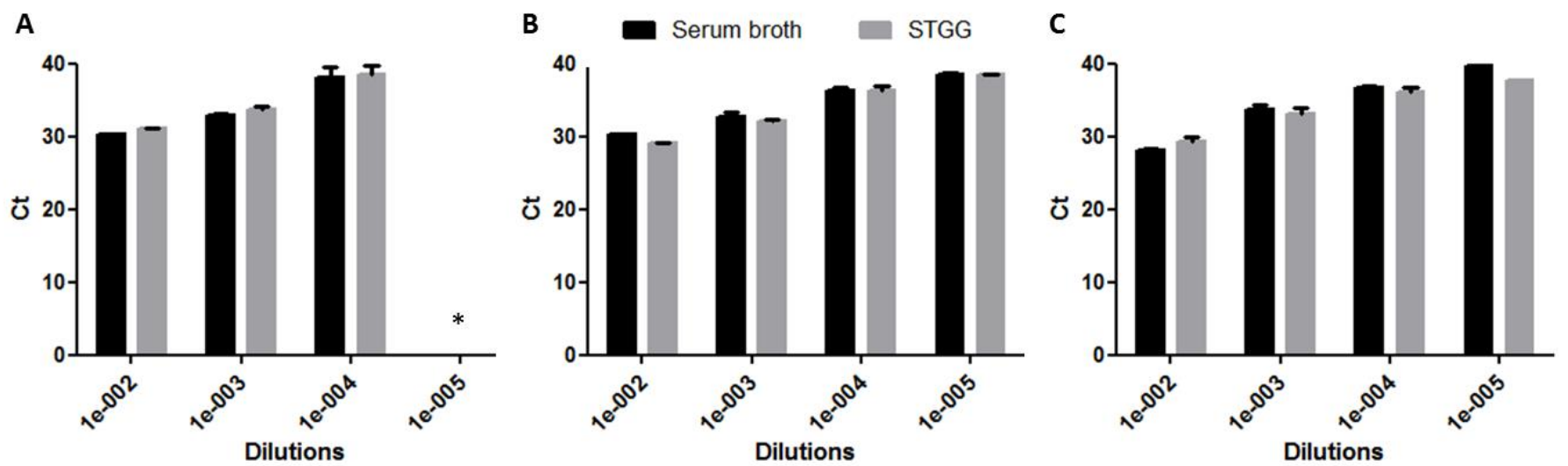

$302 *$ At a dilution of $10^{-5}$, one of the triplicates had a cycle threshold $(\mathrm{Ct})$ of 39.4 , while the other two were above 40 . This sample 303 was therefore considered negative.

306 Figure 3: Quantification of bacterial load (log counts of colony forming units [CFU]) at

307 different dilutions of enrichment broth and STGG. A: serotype 19F; B: serotype 4; C: serotype 3.

A

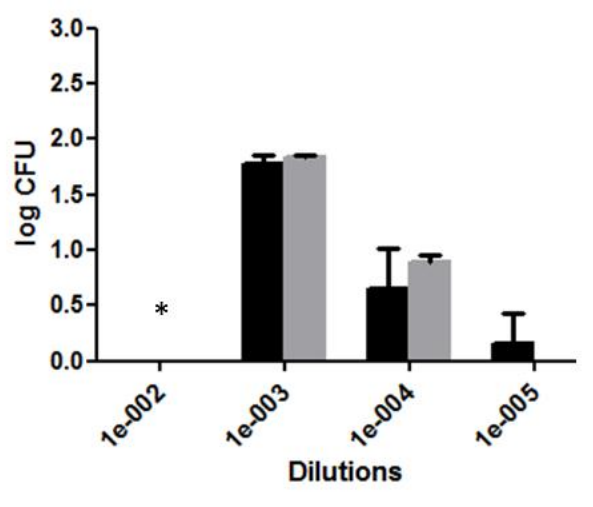

$308 *$ Uncountable.

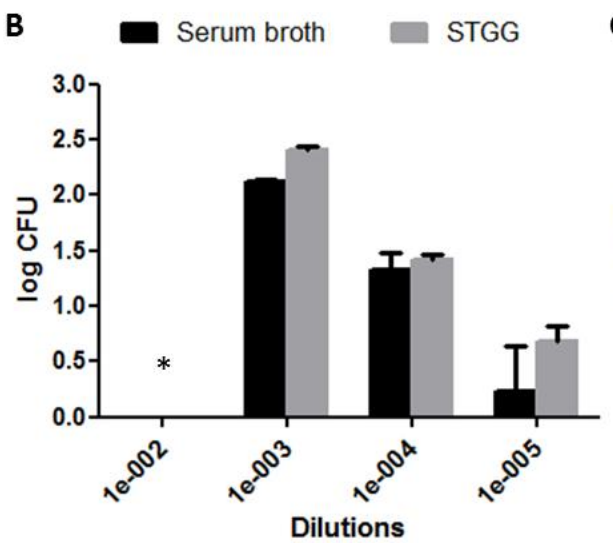

C

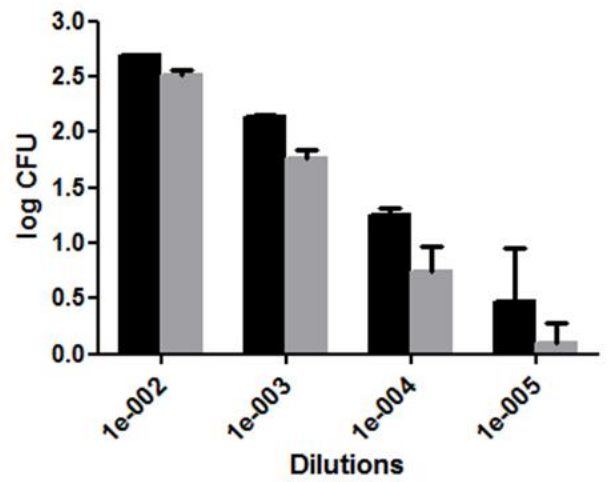

